\title{
ON CROSSED PRODUCTS OF HOPF ALGEBRAS
}

\author{
MARIA E. LORENZ AND MARTIN LORENZ
}

(Communicated by Ken Goodearl)

\begin{abstract}
Let $B=A \#_{\sigma} H$ denote a crossed product of the associative algebra $A$ with the Hopf algebra $H$. We investigate the weak dimension and the global dimension of $B$ and show that $\operatorname{wdim} B \leq \operatorname{wdim} H+w \operatorname{dim} A$ and $1 . g l d i m B \leq$ r.gldim $H+$ l.gldim $A$.
\end{abstract}

\section{INTRODUCTION}

Let $B=A \#_{\sigma} H$ denote a crossed product of the associative algebra $A$ with the Hopf algebra $H$. We establish the following estimates for the weak dimension and the global dimension of $B$ in terms of the corresponding data for $H$ and $A$ :

$$
\operatorname{wdim} B \leq \operatorname{wdim} H+w \operatorname{wim} A \quad \text { and } \quad 1 . g l d i m ~ B \leq \operatorname{rgldim} H+\operatorname{lggldim} A .
$$

The first of these estimates is a consequence of a suitable spectral sequence

$$
E_{p, q}^{2}=\operatorname{Tor}_{p}^{H}\left(k, \operatorname{Tor}_{q}^{A}(V, W)\right) \underset{p}{\Longrightarrow} \operatorname{Tor}_{n}^{B}(V, W),
$$

where $k$ is the trivial $H$-module (i.e., $H$ acts via the counit) and $V_{B}$ and ${ }_{B} W$ are arbitrary $B$-modules. This spectral sequence will be constructed in Section 2.3 along with an analogous spectral sequence for Ext which yields the estimate for global dimension. Since a ring is von Neumann regular precisely if its weak dimension is 0 , we conclude in particular that if $H$ and $A$ are both von Neumann regular, then $B$ is likewise. Specializing to the case of global dimension 0 , we also deduce the known fact that if $H$ and $A$ are both semisimple, then so is $B$ (cf. [Mont], Theorem 7.4.2). Finally, we briefly discuss relative projectivity of $B$ with respect to $A$.

Notation and basic facts. Our reference for general material about Hopf algebras are the standard texts [Abe] and [Sw]. For crossed products in particular we follow the notes [Mont]. Throughout this article, we will keep the following notation:

Received by the editors April 14, 1993; the contents of this article have been presented by the first author at the AMS Special Session "Representation theory and geometry of noncommutative algebras" in College Station, TX, on October 22, 1993.

1991 Mathematics Subject Classification. Primary 16E10, 16S40, 16W30.

Key words and phrases. Hopf algebra, crossed product, global dimension, weak dimension, projective dimension, flat dimension, von Neumann regular ring, semisimple ring.

The second author was supported in part by NSF Grant DMS-9005597. 
$k \quad$ denotes a commutative field;

$H \quad$ will be a Hopf algebra over $k$, with counit $\epsilon$; the $H$-module $k$ will always be the trivial $H$-module;

$A \quad$ denotes an associative $k$-algebra with identity 1 so that there is a weak $H$-action on $A$, denoted $(h, a) \mapsto h \cdot a \quad(h \in H, a \in A)$; $B=A \#_{\sigma} H$ will denote a crossed product, with cocycle $\sigma: H \otimes_{k} H \rightarrow A$.

Thus $B$ is an associative algebra such that there is an isomorphism of left $A$-modules

$$
A \otimes_{k} H \stackrel{\cong}{\longrightarrow} B, \quad a \otimes h \mapsto a \# h .
$$

The map $a \mapsto a \# 1$ identifies $A$ with a subalgebra of $B$. Defining a $k$-linear map $\gamma: H \rightarrow B$ by

$$
\gamma(h)=1 \# h \quad(h \in H),
$$

we have $a \gamma(h)=a \# h$ for $a \in A, h \in H$. It is known (cf. [Mont], Chapter 7) that $\gamma$ is convolution invertible and satisfies the following identities, for $h, k \in H$ and $a \in A$,

$$
\begin{aligned}
\sigma(h, k) & =\sum \gamma\left(h_{1}\right) \gamma\left(k_{1}\right) \gamma^{-1}\left(h_{2} k_{2}\right), \\
\gamma(h) \gamma(k) & =\sum \sigma\left(h_{1}, k_{1}\right) \gamma\left(h_{2} k_{2}\right), \\
\gamma^{-1}(h k) & =\sum \gamma^{-1}\left(k_{1}\right) \gamma^{-1}\left(h_{1}\right) \sigma\left(h_{2}, k_{2}\right), \\
\gamma(h) a & =\sum\left(h_{1} \cdot a\right) \gamma\left(h_{2}\right) .
\end{aligned}
$$

\section{Proofs}

2.1. Action of $H$ on homomorphisms. Let ${ }_{B} V$ and ${ }_{B} W$ be left $B$-modules. For each $\phi \in \operatorname{Hom}_{A}(V, W)$ and $h \in H$ define $\phi h: V \rightarrow W$ by

$$
(\phi h)(v)=\sum \gamma^{-1}\left(h_{1}\right) \phi\left(\gamma\left(h_{2}\right) v\right) \quad(v \in V) .
$$

Then we have the following

Lemma. The above definition makes $\operatorname{Hom}_{A}(V, W)$ a right $H$-module. There is a canonical $k$-linear isomorphism

$$
\operatorname{Hom}_{H}\left(k, \operatorname{Hom}_{A}(V, W)\right) \cong \operatorname{Hom}_{B}(V, W) .
$$

Furthermore,

$$
\operatorname{Hom}_{A}(B, W) \cong \operatorname{Hom}_{k}(H, W)
$$

as right $H$-modules (where $H$ acts on the right-hand side by $(\psi h)(k)=\psi(h k)$ for $\psi \in \operatorname{Hom}_{k}(H, W)$ and $\left.h, k \in H\right)$. Finally, if $f: V \rightarrow V^{\prime}$ and $g: W \rightarrow$ $W^{\prime}$ are B-module maps, then $g_{*} \circ f^{*}: \operatorname{Hom}_{A}\left(V^{\prime}, W\right) \rightarrow \operatorname{Hom}_{A}\left(V, W^{\prime}\right)$ is an H-module map.

Proof. The fact that $\phi h: V \rightarrow W$ is $A$-linear is proved exactly as in [Mont], proof of Theorem 7.4.2. Furthermore, the map $\operatorname{Hom}_{A}(V, W) \times H \rightarrow$ $\operatorname{Hom}_{A}(V, W),(\phi, h) \mapsto \phi h$ is clearly $k$-bilinear. Using the identities (1a) 
and (1b) we compute, for $h, k \in H$ and $v \in V$,

$$
\begin{aligned}
{[\phi(h k)](v) } & =\sum \gamma^{-1}\left(h_{1} k_{1}\right) \phi\left(\gamma\left(h_{2} k_{2}\right) v\right) \\
& =\sum \gamma^{-1}\left(k_{1}\right) \gamma^{-1}\left(h_{1}\right) \sigma\left(h_{2}, k_{2}\right) \phi\left(\gamma\left(h_{3} k_{3}\right) v\right) \\
& =\sum \gamma^{-1}\left(k_{1}\right) \gamma^{-1}\left(h_{1}\right) \phi\left[\sigma\left(h_{2}, k_{2}\right) \gamma\left(h_{3} k_{3}\right) v\right] \\
& =\sum \gamma^{-1}\left(k_{1}\right) \gamma^{-1}\left(h_{1}\right) \phi\left[\gamma\left(h_{2}\right) \gamma\left(k_{2}\right) v\right] \\
& =[(\phi h) k](v) .
\end{aligned}
$$

Thus $\operatorname{Hom}_{A}(V, W)$ is a right $H$-module.

In order to establish the first isomorphism, we first note that there is a canonical isomorphism of $\operatorname{Hom}_{H}\left(k, \operatorname{Hom}_{A}(V, W)\right)$ with the $k$-space of $H$ invariants in $\operatorname{Hom}_{A}(V, W)$, that is, with

$\operatorname{Hom}_{A}(V, W)^{H}=\left\{\phi \in \operatorname{Hom}_{A}(V, W) \mid \phi h=\epsilon(h) \phi\right.$ for all $\left.h \in H\right\}$.

Thus it suffices to show that $\operatorname{Hom}_{A}(V, W)^{H}=\operatorname{Hom}_{B}(V, W)$. Let $\phi \in$ $\operatorname{Hom}_{A}(V, W), h \in H$ and $v \in V$. Then

$$
\begin{aligned}
(\phi h)(v)=\epsilon(h) \phi(v) & \Leftrightarrow \sum \gamma^{-1}\left(h_{1}\right) \phi\left(\gamma\left(h_{2}\right) v\right)=\sum \gamma^{-1}\left(h_{1}\right) \gamma\left(h_{2}\right) \phi(v) \\
& \Leftrightarrow \phi(\gamma(h) v)=\gamma(h) \phi(v) .
\end{aligned}
$$

Since $B=A \gamma(H)$, the last condition is equivalent with $\phi \in \operatorname{Hom}_{B}(V, W)$. This proves the first isomorphism. by

Now consider the map $f: \operatorname{Hom}_{A}(B, W) \rightarrow \operatorname{Hom}_{k}(H, W)$ that is defined

$$
f(\phi)(h)=(\phi h)(1)=\sum \gamma^{-1}\left(h_{1}\right) \phi\left(\gamma\left(h_{2}\right)\right)
$$

for $\phi \in \operatorname{Hom}_{A}(B, W)$ and $h \in H$. Then $f$ is right $H$-linear. Define a map $g: \operatorname{Hom}_{k}(H, W) \rightarrow \operatorname{Hom}_{A}(B, W)$ by

$$
g(\psi)(\gamma(h))=\sum \gamma\left(h_{1}\right) \psi\left(h_{2}\right)
$$

for $\psi \in \operatorname{Hom}_{k}(H, W)$ and $h \in H$. Note that $g(\psi)$ is well defined because $B \cong A \otimes_{k} \gamma(H)$ as left $A$-modules. One readily checks that $f$ and $g$ are inverse to each other, whence the second isomorphism follows.

Finally, the last assertion is trivial and so the lemma is proved.

2.2. Action of $H$ on tensors. Let $V_{B}$ and ${ }_{B} W$ be $B$-modules. For $v \otimes w \in$ $V \otimes_{A} W$ and $h \in H$ define $h(v \otimes w) \in V \otimes_{A} W$ by

$$
h(v \otimes w)=\sum v \gamma^{-1}\left(h_{1}\right) \otimes \gamma\left(h_{2}\right) w .
$$

Using identity (2), one easily checks that this is well defined, i.e., that $h(v a \otimes w)=h(v \otimes a w)$ holds for all $v \in V, w \in W, h \in H$, and $a \in A$.

Lemma. The above definition makes $V \otimes_{A} W$ a left $H$-module. There is a canonical $k$-linear isomorphism

$$
\left.k \otimes_{H}\left(V \otimes_{A} W\right)\right) \cong V \otimes_{B} W .
$$

Furthermore,

$$
V \otimes_{A} B \cong H \otimes_{k} V
$$


as left $\mathrm{H}$-modules (where the $\mathrm{H}$-action on the right-hand side is via the action on the factor $H$. So $\left.H \otimes_{k} V \cong H^{\left(\operatorname{dim}_{k} V\right)}\right)$. Finally, if $f: V \rightarrow V^{\prime}$ and $g: W \rightarrow W^{\prime}$ are $B$-module maps, then $g \otimes f: V \otimes_{A} W \rightarrow V^{\prime} \otimes_{A} W^{\prime}$ is an H-module map. Proof. The module properties again follow readily from the identities (1a) and (1b). For the first isomorphism, note that

$$
\left.k \otimes_{H}\left(V \otimes_{A} W\right)\right) \cong V \otimes_{A} W /(\operatorname{Ker} \epsilon)\left(V \otimes_{A} W\right) .
$$

Now $(\operatorname{Ker} \epsilon)\left(V \otimes_{A} W\right)$ is the $k$-subspace of $V \otimes_{A} W$ that is generated by the elements of the form $h(v \otimes w)-\epsilon(h) v \otimes w$ for $h \in H, v \in V, w \in W$. But

$$
h(v \otimes w)-\epsilon(h) v \otimes w=\sum\left[v \gamma^{-1}\left(h_{1}\right) \otimes \gamma\left(h_{2}\right) w-v \gamma^{-1}\left(h_{1}\right) \gamma\left(h_{2}\right) \otimes w\right],
$$

and hence $(\operatorname{Ker} \epsilon)\left(V \otimes_{A} W\right)$ equals the $k$-subspace of $V \otimes_{A} W$ that is generated by the elements of the form $v \gamma(h) \otimes w-v \otimes \gamma(h) w$. Since $B=A \gamma(H)$, this proves the first isomorphism.

For the second isomorphism, define $f: H \otimes_{k} V \rightarrow V \otimes_{A} B$ by

$$
f(h \otimes v)=h(v \otimes 1)=\sum v \gamma^{-1}\left(h_{1}\right) \otimes \gamma\left(h_{2}\right) .
$$

Then $f$ is clearly $H$-linear. Furthermore, since $B \cong A \otimes_{k} \gamma(H)$ as left $A$ modules, we can define $g: V \otimes_{A} B \rightarrow H \otimes_{k} V$ by

$$
g(v \otimes \gamma(h))=\sum h_{2} \otimes v \gamma\left(h_{1}\right) .
$$

One easily checks that $f$ and $g$ are inverse to each other, and hence $f$ is an isomorphism.

The last assertion is again clear and so the lemma is proved.

2.3. Ext and Tor. The $H$-actions in Sections 2.1 and 2.2 extend to $H$ actions on Ext and Tor. We explain this for Ext, the case of Tor being entirely analogous. So let ${ }_{B} V$ and ${ }_{B} W$ be left $B$-modules and let

$$
\mathbf{P}: \cdots \stackrel{f_{n+1}}{\longrightarrow} P_{n} \stackrel{f_{n}}{\longrightarrow} \cdots \stackrel{f_{1}}{\longrightarrow} P_{0} \stackrel{f_{0}}{\longrightarrow} 0
$$

be a projective resolution of $V$, so $H_{n}(\mathbf{P})=0$ for $n \neq 0$ and $H_{0}(\mathbf{P}) \cong$ $V$. Since $B$ is projective (in fact, free) as a left $A$-module, the restriction of $\mathbf{P}$ to $A$ is a projective resolution of ${ }_{A} V$ and so we have $\operatorname{Ext}_{A}^{*}(V, W)$ $\cong H^{*}\left(\operatorname{Hom}_{A}(\mathbf{P}, W)\right)$. By Section 2.1, the components of the complex $\operatorname{Hom}_{A}(\mathbf{P}, W)$ are right $H$-modules and the differential $\left(f_{n}^{*}\right)_{n}$ is $H$-linear. Thus the cohomology $H^{*}\left(\operatorname{Hom}_{A}(\mathbf{P}, W)\right)$ is a right $H$-module and hence so is $\operatorname{Ext}_{A}^{*}(V, W)$.

Proposition. (a) Let ${ }_{B} V$ and ${ }_{B} W$ be left $B$-modules. Then there is a third quadrant spectral sequence

$$
E_{2}^{p, q}=\operatorname{Ext}_{H}^{p}\left(k, \operatorname{Ext}_{A}^{q}(V, W)\right) \underset{p}{\Longrightarrow} \operatorname{Ext}_{B}^{n}(V, W) .
$$

(b) Let $V_{B}$ and ${ }_{B} W$ be B-modules. Then there is a first quadrant spectral sequence

$$
E_{p, q}^{2}=\operatorname{Tor}_{p}^{H}\left(k, \operatorname{Tor}_{q}^{A}(V, W)\right) \underset{p}{\Longrightarrow} \operatorname{Tor}_{n}^{B}(V, W) .
$$

Proof. Both spectral sequences can be obtained as applications of the Grothendieck spectral sequence (cf. [Rot], Chapter 11$)$. We let ${ }_{B} \mathfrak{M}$ denote the category 
of left $B$-modules and similarly for the other algebras under consideration and for right modules.

(a) Let ${ }_{B} W$ be a given left $B$-module. Define functors

$$
G:{ }_{B} \mathfrak{M} \rightarrow \mathfrak{M}_{H}, \quad G(V)=\operatorname{Hom}_{A}(V, W)
$$

and

$$
F: \mathfrak{M}_{H} \rightarrow \mathfrak{M}_{k}, \quad F(X)=\operatorname{Hom}_{H}(k, X) .
$$

By Lemma 2.1, $F G$ is equivalent with the functor $\operatorname{Hom}_{B}(., W)$ and so the right derived functors $R^{n}(F G)$ are equivalent with $\operatorname{Ext}_{B}^{n}(., W)$. Moreover, if $P \in{ }_{B} \mathfrak{M}$ is projective, then $\left(R^{n} F\right)(G(P))=\operatorname{Ext}_{H}^{n}(k, G(P))=0$ for all $n>0$ and so $G(P)$ is right $F$-acyclic. Indeed, it suffices to check this equality for $P=B$. In this case, Lemma 2.1 and [Rot], Theorem 11.56 , together imply that

$$
\begin{aligned}
\operatorname{Ext}_{H}^{n}(k, G(B)) & =\operatorname{Ext}_{H}^{n}\left(k, \operatorname{Hom}_{k}(H, W)\right) \\
& \cong \operatorname{Ext}_{k}^{n}\left(k \otimes_{H} H, W\right) \\
& =\operatorname{Ext}_{k}^{n}(k, W) \\
& =0 \quad(n>0) .
\end{aligned}
$$

The required spectral sequence now follows from [Rot], Theorem 11.38.

(b) Let $V_{B}$ be a given right $B$-module. Define functors

$$
G:{ }_{B} \mathfrak{M} \rightarrow{ }_{H}^{\mathfrak{M}}, \quad G(W)=V \otimes_{A} W
$$

and

$$
F:{ }_{H} \mathfrak{M} \rightarrow{ }_{k} \mathfrak{M}, \quad F(X)=k \otimes_{H} X .
$$

By Lemma 2.2, $F G$ is equivalent with the functor $V \otimes_{B}($.$) and so the left de-$ rived functors $L_{n}(F G)$ are equivalent with $\operatorname{Tor}_{n}^{B}(V,$.$) . Furthermore, Lemma$ 2.2 implies that $G$ maps projective $B$-modules to projective $H$-modules. Since projective $H$-modules are left $F$-acyclic, the required spectral sequence follows from [Rot], Theorem 11.39.

2.4. Homological dimension. The above proposition directly implies the following estimates for the flat dimension and the projective dimension of modules, denoted fdim and pdim, respectively.

Corollary. (a) Let ${ }_{B} V$ be a B-module. Then $\operatorname{pdim}_{B} V \leq \operatorname{pdim} k_{H}+\operatorname{pdim}_{A} V$. Consequently, l.gldim $B \leq \mathrm{r} . g l d i m ~ H+\operatorname{lgldim} A$. In particular, if $A$ and $H$ are both semisimple (gldim 0 ), then so is $B$ (cf. [Mont], Theorem 7.4.2).

(b) Let $V_{B}$ be a B-module. Then $\operatorname{fdim} V_{B} \leq \operatorname{fdim} k_{H}+\mathrm{fdim} V_{A}$. Therefore, $\operatorname{wdim} B \leq \operatorname{wdim} H+w \operatorname{wdim} A$. In particular, if $A$ and $H$ are both von Neumann regular (wdim 0 ), then so is $B$.

We note that

$$
\text { r.gldim } H=\operatorname{pdim} k_{H} \quad \text { and } \quad \operatorname{wdim} H=\operatorname{fdim} k_{H} .
$$

For, if $\mathbf{P}$ is a projective resolution of $k_{H}$, then, for any right $H$-module $X$, $X \otimes_{k} \mathbf{P}$ is a resolution of $X \otimes_{k} k \cong X$ which consists of projective $H$-modules. To see the latter, note that the Fundamental Theorem of Hopf modules ([Mont], Theorem 1.9.4) implies that $X \otimes_{k} H$ is a free $H$-module, and hence $X \otimes_{k} P$ is projective for any projective $H$-module $P$. Thus $\operatorname{pdim} X_{H} \leq \operatorname{pdim} k_{H}$ which proves the first equality. For the second equality, consider a flat resolution $\mathbf{P}$ of $k_{H}$ and use the fact that flat modules are direct limits of free modules to obtain that $X \otimes_{k} \mathbf{P}$ is a flat resolution of $X$. 
2.5. Relative projectivity. Recall that if $R \subseteq S$ is a pair of rings, then $S$ is called projective relative to $R$ (or $R$-projective) if the following holds: Given $S$ modules ${ }_{S} W \subseteq{ }_{S} V$ so that $W$ is a direct summand of $V$ as $R$-modules, then $W$ is a direct summand of $V$ as $S$-modules. The following result is identical with [Mont], Theorem 7.4.2(1), but the proof below is a nice application of the techniques of Section 2.1 .

Corollary. If $H$ is semisimple, then $B$ is projective relative to $A$.

Proof. First note that if $f: X \rightarrow Y$ is an epimorphism in $\mathfrak{M}_{H}$ then $f\left(X^{H}\right)=$ $Y^{H}$, because $f$ splits by assumption on $H$. Now let ${ }_{B} W \subseteq{ }_{B} V$ so that $W$ is a direct summand of $V$ as $A$-modules. Then the canonical epimorphism $\pi$ : $V \rightarrow V / W$ splits in $A_{A} \mathfrak{M}$ and so the map $\pi_{*}: \operatorname{Hom}_{A}(V / W, V) \rightarrow \operatorname{End}_{A}(V / W)$ is surjective. Since $\pi_{*}$ is a map in $\mathfrak{M}_{H}$, we deduce from the foregoing that $\pi_{*}\left(\operatorname{Hom}_{A}(V / W, V)^{H}\right)=\operatorname{End}_{A}(V / W)^{H}$. By Section 2.1, $\operatorname{Hom}_{A}(V / W, V)^{H}=$ $\operatorname{Hom}_{B}(V / W, V)$ and $\operatorname{End}_{A}(V / W)^{H}=\operatorname{End}_{B}(V / W)$. Thus there exists $\mu \in$ $\operatorname{Hom}_{B}(V / W, V)$ with $\pi_{*}(\mu)=\pi \circ \mu=\operatorname{Id}_{V / W}$ and so $W$ is a direct summand of $V$ as $B$-modules, as required.

\section{REFERENCES}

[Abe] E. Abe, Hopf algebras, Cambridge Univ. Press, Cambridge, 1977.

[Mont] S. Montgomery, Hopf algebras and their actions on rings, preprint, 1993.

[Rot] J. Rotman, An introduction to homological algebra, Academic Press, Orlando, 1979.

[Sw] M. Sweedler, Hopf algebras, Benjamin, New York, 1969.

Department of Mathematics, University of Pittsburgh, Pittsburgh, Pennsylvania 15260

E-mail address: angelone@mern.math.pitt.edu

Department of Mathematics, Temple University, Philadelphia, Pennsylvania 19122

E-mail address: lorenz@math. temple.edu 